\title{
Anti-EpCAM monoclonal antibody exerts antitumor activity against oral squamous cell carcinomas
}

\author{
MIKA K. KANEKO ${ }^{1 *}$, TOMOKAZU OHISHI ${ }^{2 *}$, JUNKO TAKEI $^{1,3^{*}}$, MASATO SANO $^{1}$, \\ TAKURO NAKAMURA ${ }^{1}$, HIDEKI HOSONO ${ }^{1}$, MIYUKI YANAKA ${ }^{1}$, TEIZO ASANO ${ }^{1}$, \\ YUSUKE SAYAMA $^{1}$, HIROYUKI HARADA ${ }^{3}$, MANABU KAWADA $^{2}$ and YUKINARI KATO ${ }^{1,4}$
}

${ }^{1}$ Department of Antibody Drug Development, Tohoku University Graduate School of Medicine, Aoba-ku, Sendai, Miyagi 980-8575; ${ }^{2}$ Institute of Microbial Chemistry (BIKAKEN), Numazu, Microbial Chemistry Research Foundation, Numazu-shi, Shizuoka 410-0301; ${ }^{3}$ Department of Oral and Maxillofacial Surgery, Graduate School of Medical and

Dental Sciences, Tokyo Medical and Dental University, Bunkyo-ku, Tokyo 113-8510;

${ }^{4}$ New Industry Creation Hatchery Center, Tohoku University, Aoba-ku, Sendai, Miyagi 980-8575, Japan

Received July 14, 2020; Accepted August 28, 2020

DOI: 10.3892/or.2020.7808

\begin{abstract}
The epithelial cell adhesion molecule (EpCAM) is a calcium-independent, homophilic, intercellular adhesion factor classified as a transmembrane glycoprotein. In addition to cell adhesion, EpCAM also contributes to cell signaling, differentiation, proliferation, and migration. EpCAM is an essential factor in the carcinogenesis of numerous human cancers. In the present study, we developed and validated an anti-EpCAM monoclonal antibody (mAb), EpMab-16 ( $\operatorname{IgG}_{2 a}$, kappa), by immunizing mice with EpCAM-overexpressing CHO-K1 cells. EpMab-16 specifically reacted with endogenous EpCAM in oral squamous cell carcinoma (OSCC) cell lines in flow cytometry and Western blot analyses. It exhibited a plasma membrane-like stain pattern in OSCC tissues upon immunohistochemical analysis. The $K_{\mathrm{D}}$ for EpMab-16 in SAS and HSC-2 OSCC cells were assessed via flow cytometry at $1.1 \times 10^{-8}$ and $1.9 \times 10^{-8} \mathrm{M}$, respectively, suggesting moderate binding affinity of EpMab-16 for EpCAM. We then assessed
\end{abstract}

Correspondence to: Professor Yukinari Kato, New Industry Creation Hatchery Center, Tohoku University, 2-1 Seiryo-machi, Aoba-ku, Sendai, Miyagi 980-8575, Japan

E-mail: yukinarikato@med.tohoku.ac.jp

*Contributed equally

Abbreviations: ADCC, antibody-dependent cellular cytotoxicity; BSA, bovine serum albumin; CBIS, Cell-Based Immunization and Screening; CDC, complement-dependent cytotoxicity; DMEM, Dulbecco's modified Eagle's medium; EDTA, ethylenediaminetetraacetic acid; FBS, fetal bovine serum; mAb, monoclonal antibody; OSCC, oral squamous cell carcinoma; PBS, phosphate-buffered saline

Key words: EpCAM, monoclonal antibody, ADCC, CDC, antitumor activity, oral cancer whether the EpMab-16 induced antibody-dependent cellular cytotoxicity (ADCC) and complement-dependent cytotoxicity (CDC) against OSCC cell lines, and antitumor capacity in a murine xenograft model. In vitro experiments revealed strong ADCC and CDC inducement against OSCC cells treated with EpMab-16. In vivo experiments on OSCC xenografts revealed that EpMab-16 treatment significantly reduced tumor growth compared with the control mouse IgG. These data indicated that EpMab-16 could be a promising treatment option for EpCAM-expressing OSCCs.

\section{Introduction}

Cellular junctions are critical for maintaining cellular architecture and are comprised of several different cell adhesion molecules (CAM) (1). Thus, the four major CAM families are selectins, cadherins, integrins, and the immunoglobulin CAM superfamily (2). The extracellular domain of selectins consists of a calcium-dependent lectin domain, an epidermal growth factor (EGF)-like domain, a domain homologous to EGF, and two to nine consensus repeats. Selectins also contain a hydrophobic transmembrane domain and a short cytoplasmic tail. The cadherins are calcium-dependent glycoproteins that have an extracellular domain CAM with three to five internal repeats, along with a single-span transmembrane domain and an intracellular domain (2). Integrins are composed of two or more noncovalently associated membrane-spanning subunits labeled $\alpha$ and $\beta$ (3). The specific combination of $\alpha$ and $\beta$ subunits confers specificity for different extracellular ligands and their concomitant intracellular signaling events, and each represents a significant receptor family within the context of interaction with the extracellular matrix (3). The Ig-CAMs are calcium-independent, with an extracellular domain comprising a ligand-binding region of four to six Ig-like repeats, one to five fibronectin-like repeats, a transmembrane domain, and an intracellular component (1). While these families predominate, numerous CAMs do not share any structural similarities with them, one of which is epithelial cell adhesion molecule (EpCAM) (4). 
EpCAM was discovered approximately 40 years ago and was one of the first identified human tumor-associated biomarkers (5). These days, EpCAM is also considered as a marker for tumor-initiating cells (6). EpCAM is a transmembrane, calcium-independent, homophilic, intercellular adhesion glycoprotein. It has three distinct domains, extracellular, transmembrane, and intracellular. In addition to cell adhesion, EpCAM functions include cell signaling, differentiation, proliferation, and migration (4). EpCAM has been implicated in carcinogenesis and is expressed robustly in numerous human epithelial cancers, including cancers of the lung, colon, breast, ovary, cervix, and oral cavity, making it a promising target for cancer diagnosis and therapy (7-9).

Oral cancers account for approximately $2 \%$ of all cancer cases worldwide (10). More than 350,000 individuals are diagnosed with oral cancer each year, and it is ultimately fatal in nearly half of all cases $(11,12)$. Of the defined histological types of oral cancers, $>90 \%$ of patients develop oral squamous cell carcinoma (OSCC) on the lips or within the oral cavity (13). The most effective current treatments for OSCC vary. Stage-I and -II OSCCs are treated with surgery or radiotherapy; advanced stage-III and -IV disease are treated with a combination of excision, radiation, and chemotherapy (14). Typically, chemotherapeutic regimens include cisplatin as a first-line agent; it is often combined with docetaxel or 5-fluorouracil $(15,16)$. Other anticancer drugs, such as paclitaxel, methotrexate, and carboplatin, have also been used for OSCCs (17). Effective molecular-targeting drugs, however, including antibody therapies, remain lacking.

In the present study, we developed a set of new anti-EpCAM monoclonal antibodies (mAbs), using Cell-Based Immunization and Screening (CBIS) methods (18). We then tested whether these anti-EpCAM mAbs induced antibody-dependent cellular cytotoxicity (ADCC), complement-dependent cytotoxicity (CDC), or other antitumor activity against oral cancers in a murine xenograft model.

\section{Materials and methods}

Plasmids. The Genome Network Project clone IRAK021G03 (EpCAM) was provided by the RIKEN BioResource Research Center through the National BioResource Project of the MEXT and AMED agencies of Japan (19-22). EpCAM DNA plus a $\mathrm{C}$-terminal PA tag that is recognized by the anti-PA tag $\mathrm{mAb}$ (NZ-1), was subcloned into a pCAG-Ble vector (FUJIFILM Wako Pure Chemical Corporation).

Cell lines. P3X63Ag8U.1 (P3U1) and CHO-K1 cells were obtained from the American Type Culture Collection (ATCC). CHO/EpCAM was established by transfecting pCAG/EpCAM-PA into CHO-K1 cells using the Neon Transfection System (Thermo Fisher Scientific, Inc.). A few days after transfection, cells positive for anti-EpCAM mAb (clone 9C4; cat. no. 324202; BioLegend, Inc.) were sorted using a cell sorter (SH800; Sony Biotecnology Corp.), and stable transfectants were cultured at $37^{\circ} \mathrm{C}$ for 14 days on media containing $0.5 \mathrm{mg} / \mathrm{ml}$ zeocin (InvivoGen). OSCC cell lines, including SAS (tongue) and HSC-2 (oral cavity), were obtained from the Japanese Collection of Research Bioresources Cell Bank. P3U1, CHO-K1 and CHO/EpCAM were cultured in
RPMI-1640 media (Nacalai Tesque, Inc.). SAS and HSC-2 were cultured in Dulbecco's modified Eagle's medium (DMEM; Nacalai Tesque, Inc.). The media were supplemented with $10 \%$ heat-inactivated fetal bovine serum (FBS; Thermo Fisher Scientific Inc.), $100 \mathrm{U} / \mathrm{ml}$ of penicillin (Nacalai Tesque, Inc.), $100 \mu \mathrm{g} / \mathrm{ml}$ streptomycin (Nacalai Tesque, Inc.), and $0.25 \mu \mathrm{g} / \mathrm{ml}$ amphotericin B (Nacalai Tesque, Inc.), and incubated at $37^{\circ} \mathrm{C}$ for 14 days in a humidified atmosphere containing $5 \% \mathrm{CO}_{2}$.

Antibodies. Purified mouse IgG (cat. no. I8765) and mouse $\mathrm{IgG}_{2 \mathrm{a}}$ (cat. no. M7769) were purchased from Sigma-Aldrich; Merck KGaA. Anti-EpCAM mAbs were purified using Protein G-Sepharose (GE Healthcare Bio-Sciences).

Animals. Thirty-eight 5-week-old female BALB/c nude mice (mean weight, $15 \pm 3 \mathrm{~g}$ ) were purchased from Charles River Laboratories, Inc. All animal experiments were performed in accordance with institutional guidelines and regulations to minimize animal suffering and distress in the laboratory. The Institutional Committee for experiments of the Institute of Microbial Chemistry (Permit no. 2019-066) approved the animal studies for ADCC and antitumor activity. Mice were maintained in a specific pathogen-free environment on an 11-h light/13-h dark cycle at a temperature of $23 \pm 2^{\circ} \mathrm{C}$ and $55 \pm 5 \%$ humidity with food and water supplied ad libitum throughout the experiments. Mice were monitored for health and weight every 1 or 4 days. Experiments on mice were conducted in three or fewer weeks. Weight loss exceeding $25 \%$ or tumor size exceeding $3,000 \mathrm{~mm}^{3}$ were identified as humane endpoints for euthanasia. At humane and experimental endpoints, mice were euthanized by cervical dislocation, and death was verified by validating respiratory and cardiac arrest.

Hybridoma production. We used a CBIS method (18) to develop mAbs against EpCAM. Briefly, one BALB/c mouse was intraperitoneally (i.p.) immunized with $\mathrm{CHO} / \mathrm{EpCAM}$ cells $\left(1 \times 10^{8}\right)$ along with Imject Alum adjuvant (Thermo Fisher Scientific, Inc.). The procedure included three additional immunizations, followed by a final booster injection administered i.p. two days before spleen cell harvesting. Spleen cells were then fused with P3U1 cells using PEG1500 (Roche Diagnostics). Hybridomas were grown at $37^{\circ} \mathrm{C}$ for 10 days in RPMI-1640 media with HAT Supplement (50x) (cat. no. 21060017; Thermo Fisher Scientific, Inc.) for selection. Culture supernatants were screened using flow cytometry.

Flow cytometry. Cells $\left(2 \times 10^{5}\right.$ cells $\left./ \mathrm{ml}\right)$ were harvested after brief exposure to $0.25 \%$ trypsin in $1 \mathrm{mM}$ ethylenediaminetetraacetic acid (EDTA; Nacalai Tesque, Inc.). After washing with $0.1 \%$ bovine serum albumin (BSA; Nacalai Tesque, Inc.) in phosphate-buffered saline (PBS; Nacalai Tesque, Inc.), cells were treated with $1 \mu \mathrm{g} / \mathrm{ml}$ of anti-EpCAM mAbs for $30 \mathrm{~min}$ at $4^{\circ} \mathrm{C}$, and then with Alexa Fluor 488-conjugated anti-mouse IgG (1:1,000; product no. 4408; Cell Signaling Technology, Inc.). Fluorescence data were collected using an EC800 Cell Analyzer (Sony Biotechnology Corp.).

Western blot analyses. Cell pellets were resuspended in PBS with 1\% Triton X-100 (cat. no. 168-11805; FUJIFILM Wako Pure Chemical Corporation) and $50 \mu \mathrm{g} / \mathrm{ml}$ aprotinin 
(product no. 03346-84; Nacalai Tesque, Inc.). Protein concentration was determined by BCA method. Cell lysates were boiled in sodium dodecyl sulfate sample buffer (Nacalai Tesque, Inc.). The samples (10 $\mu \mathrm{g} /$ lane) were then electrophoresed on 5-20\% polyacrylamide gels (Nacalai Tesque, Inc.) and transferred onto polyvinylidene difluoride (PVDF) membranes (Merck KGaA). After blocking with $4 \%$ skim milk (Nacalai Tesque, Inc.) for $1 \mathrm{~h}$, the membrane was incubated with an anti-EpCAM mAb $(5 \mu \mathrm{g} / \mathrm{ml})$ or anti- $\beta$-actin $(1 \mu \mathrm{g} / \mathrm{ml}$; clone AC-15; cat. no. A5441; Sigma-Aldrich; Merck KGaA) for $1 \mathrm{~h}$, followed by incubation with HRP-conjugated anti-mouse immunoglobulins (cat. no. P0260; Agilent Technologies, Inc.) or anti-rat IgG (cat. no. A9542; Sigma-Aldrich; Merck KGaA) at a 1:2,000 dilution for $1 \mathrm{~h}$. The membrane was developed using the ImmunoStar LD Chemiluminescence Reagent (FUJIFILM Wako Pure Chemical Corporation) and a Sayaca-Imager (DRC Co., Ltd.). All western blot procedures were performed at room temperature.

Immunohistochemical analyses. Histologic sections 4- $\mu \mathrm{m}$ thick of an oral cancer tissue array (cat. no. OR481; US Biomax, Inc.) were autoclaved directly in EnVision FLEX Target Retrieval Solution, High pH (Agilent Technologies, Inc.) for $20 \mathrm{~min}$. Sections were then incubated with $10 \mu \mathrm{g} / \mathrm{ml}$ of an anti-EpCAM mAb for $1 \mathrm{~h}$ at room temperature and treated using an Envision+ kit (Agilent Technologies, Inc.) for $30 \mathrm{~min}$ according to the manufacturer's instructions. The color was developed using 3, 3'-diaminobenzidine tetrahydrochloride (DAB; Agilent Technologies Inc.) for $2 \mathrm{~min}$ at room temperature, and sections were then counterstained with hematoxylin (FUJIFILM Wako Pure Chemical Corporation) at room temperature for $5 \mathrm{~min}$. Hematoxylin and eosin (H\&E) staining (FUJIFILM Wako Pure Chemical Corporation) was performed using consecutive tissue sections at room temperature for 5 min. Leica DMD108 (Leica Microsystems GmbH) was used to examine the sections and obtain images (x100 and x400).

Determination of the binding affinity. Cells $\left(2 \times 10^{5} \mathrm{cells} / \mathrm{ml}\right)$ were suspended in $100 \mu \mathrm{l}$ of serially diluted anti-EpCAM $\mathrm{mAb}(6 \mathrm{ng} / \mathrm{ml}-100 \mu \mathrm{g} / \mathrm{ml})$, followed by the addition of Alexa Fluor 488-conjugated anti-mouse IgG (1:200; cat. no. 4408; Cell Signaling Technology, Inc.). Fluorescence data were collected using an EC800 Cell Analyzer (Sony Biotechnology Corp.). The dissociation constant $\left(K_{\mathrm{D}}\right)$ was calculated by fitting binding isotherms to built-in, one-site binding models in GraphPad Prism 6 (GraphPad Software, Inc.).

$A D C C$. ADCC inducement by EpCAM was assayed as follows. Six female five-week-old $\mathrm{BALB} / \mathrm{c}$ nude mice (mean weight, $15 \pm 3 \mathrm{~g}$ ) were purchased from Charles River Laboratories, Inc. After euthanasia by cervical dislocation, spleens were removed aseptically, and single-cell suspensions were obtained by forcing spleen tissues through a sterile cell strainer (product no. 352360; BD Falcon; Corning, Inc.) with a syringe. Erythrocytes were lysed with a 10-sec exposure to ice-cold distilled water. The splenocytes were washed with DMEM and resuspended in DMEM with 10\% FBS; this preparation was designated as effector cells. The target tumor cells were labeled with $10 \mu \mathrm{g} / \mathrm{ml}$ Calcein-AM (Thermo Fisher Scientific, Inc.) and resuspended in the same medium. The target cells were transferred to 96 -well plates, at $2 \times 10^{4}$ cells/well, and mixed with effector cells at an effector-to-target ratio of 50:1, along with $100 \mu \mathrm{g} / \mathrm{ml}$ of anti-EpCAM antibodies or control mouse $\mathrm{IgG}_{2 \mathrm{a}}$. After a 4-h incubation at $37^{\circ} \mathrm{C}$, Calcein-AM release into the supernatant was measured for each well. Fluorescence intensity was assessed using a microplate reader (Power Scan HT; BioTek Instruments, Inc.) with an excitation wavelength of $485 \mathrm{~nm}$ and an emission wavelength of $538 \mathrm{~nm}$. Cytolytic activity was measured as a percentage of lysis and calculated using the equation: Percentage of lysis $(\%)=(\mathrm{E}-\mathrm{S}) /(\mathrm{M}-\mathrm{S}) \mathrm{x} 100$, where $\mathrm{E}$ is the fluorescence measured in combined cultures of target and effector cells, $\mathrm{S}$ is the spontaneous fluorescence of the target cells, and $\mathrm{M}$ is the maximum fluorescence measured after lysis of all cells with buffer containing $0.5 \%$ Triton $\mathrm{X}-100$, $10 \mathrm{mM}$ Tris- $\mathrm{HCl}$ (pH 7.4), and 10 mM EDTA.

$C D C$. CDC inducement by EpCAM was assayed as follows. Target cells were labeled with $10 \mu \mathrm{g} / \mathrm{ml}$ Calcein-AM (Thermo Fisher Scientific, Inc.) and resuspended in medium. Target cells were plated in 96 -well plates, at $2 \times 10^{4}$ cells/well, and $10 \%$ rabbit complement (Low-Tox-M rabbit complement; Cedarlane Laboratories), $100 \mu \mathrm{g} / \mathrm{ml}$ of anti-EpCAM antibodies, or control $\mathrm{IgG}$ (mouse $\mathrm{IgG}_{2 \mathrm{a}}$ ) was added to each well. After $4 \mathrm{~h}$ of incubation at $37^{\circ} \mathrm{C}$, Calcein-AM release into the supernatant was measured for each well. Fluorescence intensity was calculated as described in the ADCC section above.

Antitumor activity of anti-EpCAM mAbs in xenografts of oral cancers. Thirty-two five-week-old female BALB/c nude mice were purchased from Charles River Laboratories Japan, Inc. After a two-week acclimation period, these mice were used in experiments at seven weeks of age (mean weight, $16 \pm 2 \mathrm{~g})$. HSC-2 and SAS cells $\left(0.3 \mathrm{ml}\right.$ of $1.33 \times 10^{8}$ cells $/ \mathrm{ml}$ in DMEM) were mixed with $0.5 \mathrm{ml} \mathrm{BD}$ Matrigel Matrix Growth Factor Reduced (BD Biosciences), and $100 \mu \mathrm{l}$ of this suspension $\left(5 \times 10^{6}\right.$ cells) was injected subcutaneously into the left flank of each animal. On day 1 post-inoculation, $100 \mu \mathrm{g}$ of an anti-EpCAM mAb or control mouse IgG in $100 \mu \mathrm{l}$ PBS was injected i.p. Additional antibody inoculations were performed on days 8 and 14. Seventeen days or eighteen days after cell implantation, all mice were euthanized by cervical dislocation, and tumor diameters and volumes were measured and recorded.

Statistical analyses. All data are expressed as mean \pm SEM. Statistical analysis was conducted with one-way ANOVA and Tukey's multiple comparisons tests for ADCC and CDC, one-way ANOVA and Sidak's multiple comparisons tests for tumor volume and mouse weight, and Welch's t-test for tumor weight. All calculations were performed with GraphPad Prism 7 (GraphPad Software, Inc.). A P-value of $<0.05$ was considered to indicate a statistically significant difference.

\section{Results}

Development and characterization of anti-EpCAM $m A b s$. Anti-EpCAM mAbs were developed by immunizing a single mouse with $\mathrm{CHO} / \mathrm{EpCAM}$ cells. Hybridomas were cultured, and supernatants positive for $\mathrm{CHO} / \mathrm{EpCAM}$ and negative for 

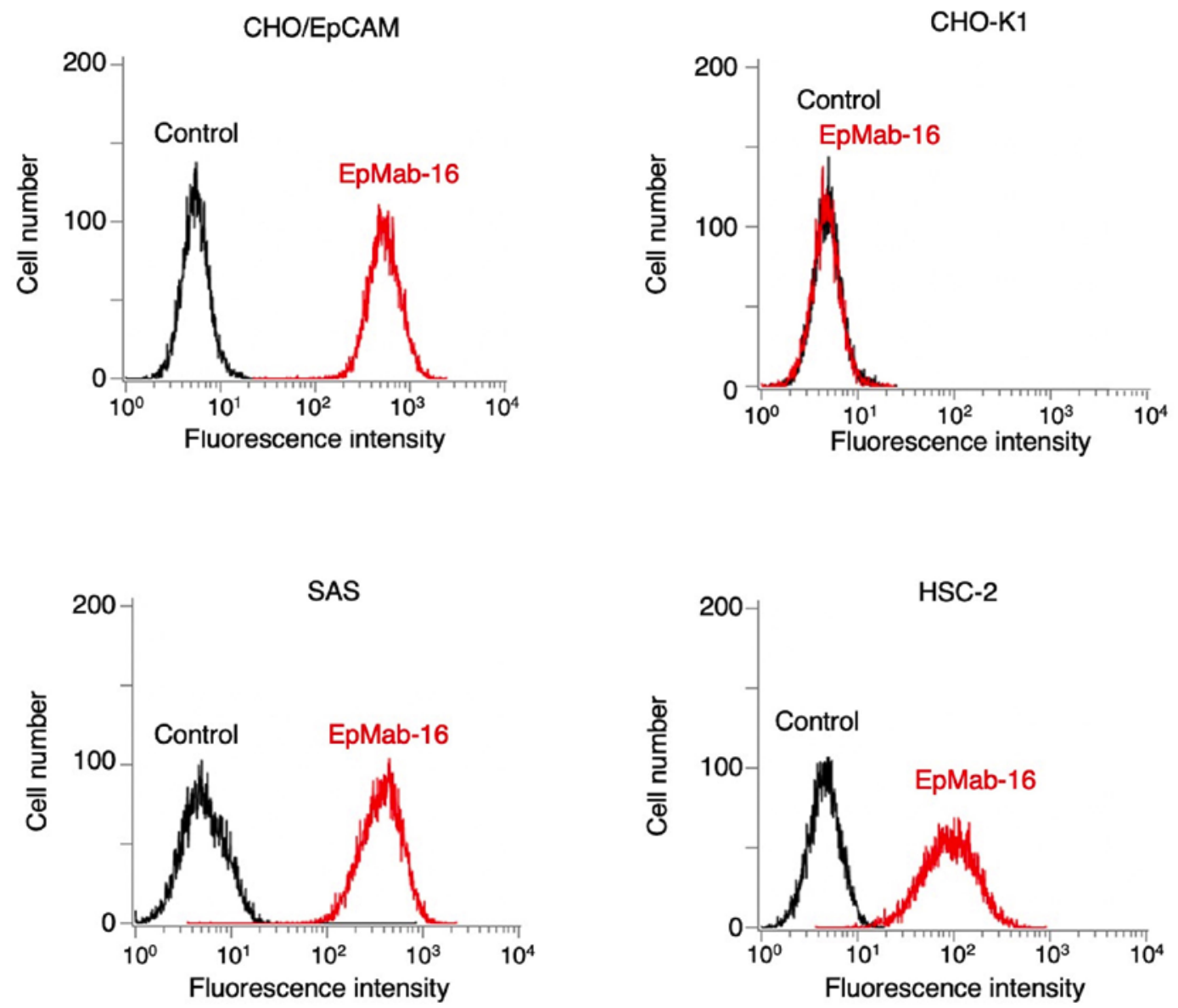

Figure 1. Recognition of EpCAM by an anti-EpCAM mAb. CHO/EpCAM, CHO-K1, SAS, and HSC-2 cells were treated with EpMab-16 (1 $\mu \mathrm{g} / \mathrm{ml})$ and buffer control, followed by secondary antibodies. EpCAM, epithelial cell adhesion molecule.

CHO-K1 were selected by flow cytometry. Further screening using western blot and immunohistochemical assays were performed for validation, resulting in EpMab-16 ( $\mathrm{IgG}_{2 \mathrm{a}}$, kappa) identification and establishment.

Flow cytometry was then used to assess the sensitivity of EpMab-16 in CHO/EpCAM and OSCC cell lines (SAS and HSC-2). EpMab-16 reacted with CHO/EpCAM cells but not with CHO-K1 cells (Fig. 1). EpMab-16 also reacted with SAS and HSC-2 cells (Fig. 1). Flow cytometric data indicated that EpMab-16 was highly sensitive and highly specific for EpCAM.

Then a western blot assay was conducted to delineate the sensitivity of EpMab-16 further. Lysates of $\mathrm{CHO} / \mathrm{EpCAM}$, SAS, and HSC-2 cells were probed, and the results revealed that EpMab-16 detected $35 \mathrm{kDa}$ band of EpCAM strongly in cell lysates from CHO/EpCAM, whereas it detected EpCAM moderately in cell lysates from SAS, and faintly from HSC-2 cells, indicating that EpMab-16 could detect both exogenous and endogenous EpCAM (Fig. 2).

Immunohistochemical analysis results revealed that EpMab-16 detected membrane antigens in oral cancer tissues (Fig. 3A, B, E and F). Among 38 OSCC cases in the oral cancer tissue array, 6 cases (16\%) were stained by EpMab-16. In this staining, antigen retrieval using EnVision FLEX Target Retrieval Solution High $\mathrm{pH}$ was performed. The signal was lower for citrate buffer ( $\mathrm{pH}$ 6.0) antigen retrieval (data not shown), such that antigen retrieval using high $\mathrm{pH}$ was deemed

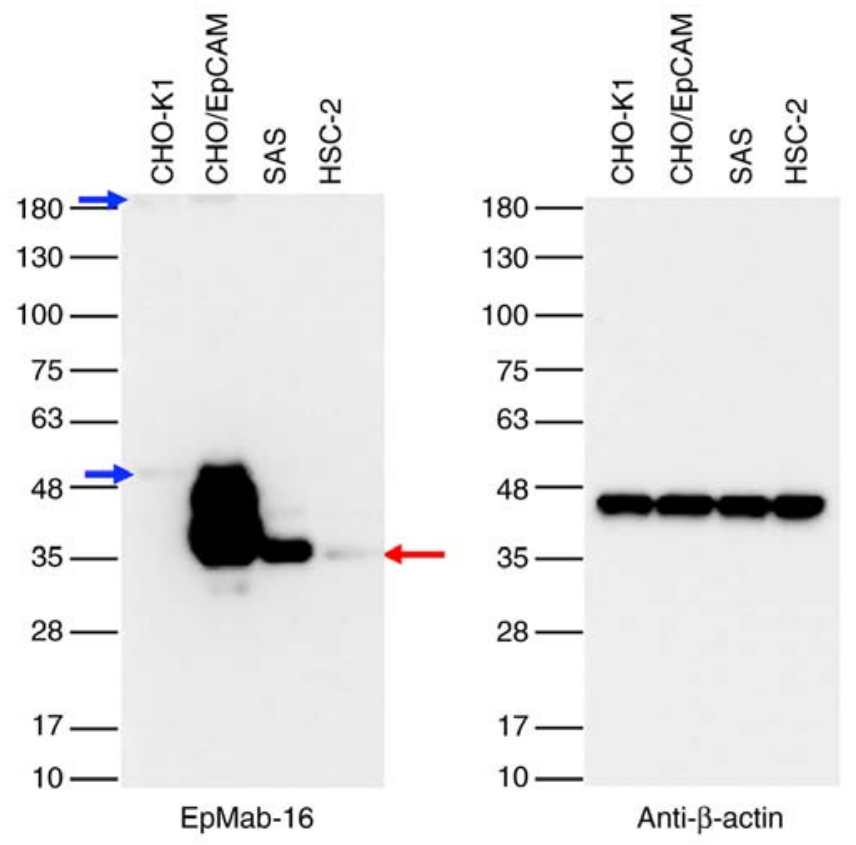

Figure 2. Western blot analysis using an anti-EpCAM mAb. Cell lysates $(10 \mu \mathrm{g})$ of CHO-K1, CHO/EpCAM, SAS, and HSC-2 were electrophoresed and transferred onto PVDF membranes. The membranes were incubated with $5 \mu \mathrm{g} / \mathrm{ml}$ of EpMab-16 and $1 \mu \mathrm{g} / \mathrm{ml}$ of anti- $\beta$-actin, followed by peroxidase-conjugated anti-mouse immunoglobulins. The red arrow indicates EpCAM. The blue arrows indicate non-specific bands, which were detected in both $\mathrm{CHO}-\mathrm{K} 1$ and $\mathrm{CHO} / \mathrm{EpCAM}$ cells. EpCAM, epithelial cell adhesion molecule; mAb, monoclonal antibody; PVDF, polyvinylidene difluoride. 


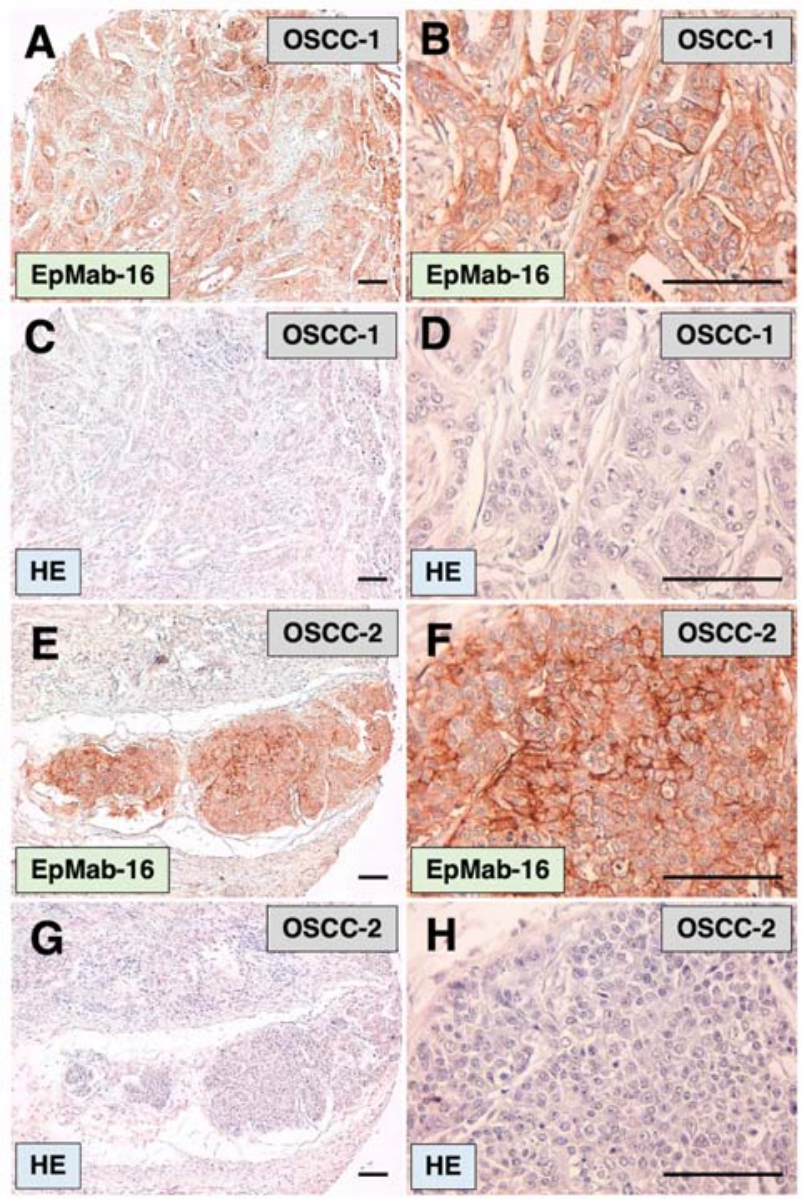

Figure 3. Immunohistochemical analyses of OSCC tissues using EpMab-16. (A and B) FFPE tissue sections of OSCC (case 1) were incubated with $10 \mu \mathrm{g} / \mathrm{ml}$ of EpMab-16. (C and D) H\&E staining against consecutive OSCC tissues (case 1). (E and F) FFPE tissue sections of OSCC (case 2) were incubated with $10 \mu \mathrm{g} / \mathrm{ml}$ of EpMab-16. ( $\mathrm{G}$ and $\mathrm{H}$ ) $\mathrm{H} \& \mathrm{E}$ staining against consecutive OSCC tissues (case 2). Scale bar, $100 \mu \mathrm{m}$. OSCC, oral squamous cell carcinoma; FFPE, formalin-fixed paraffin-embedded; H\&E, hematoxylin and eosin.

essential for immunostaining using EpMab-16. Hematoxylin and eosin (H\&E) staining was performed using consecutive OSCC tissues (Fig. 3C, D, G and H). Results indicated that EpMab-16 detected EpCAM in immunohistochemical analysis on formalin-fixed paraffin-embedded (FFPE) tissues effectively.

Then a kinetic analysis of the interactions of EpMab-16 with SAS and HSC-2 oral cancer cell lines was then conducted using flow cytometry. The $K_{\mathrm{D}}$ for EpMab-16 in SAS cells was $1.1 \times 10^{-8} \mathrm{M}$, and the $K_{\mathrm{D}}$ for EpMab-16 against HSC-2 cells was $1.9 \times 10^{-8} \mathrm{M}$ (Fig. 4), indicating a moderate binding affinity of EpMab-16 against oral cancer cells.

$A D C C$ and $C D C$ activities of EpMab-16 in oral cancer cell lines. We then examined whether EpMab-16 (mouse $\mathrm{IgG}_{2 \mathrm{a}}$ ) induced ADCC and CDC antitumor action in EpCAM-expressing SAS and HSC-2 oral cancer cell lines. EpMab-16 exhibited higher ADCC (48\% cytotoxicity) in SAS cells than that of control mouse $\operatorname{IgG}_{2 \mathrm{a}}(11 \%$ cytotoxicity; $\mathrm{P}<0.01)$ or control PBS $(9.4 \%$ cytotoxicity; $\mathrm{P}<0.01)$ treatment (Fig. 5A). Similarly, EpMab-16 exhibited higher ADCC (27\% cytotoxicity) in HSC-2 cells than that of control mouse
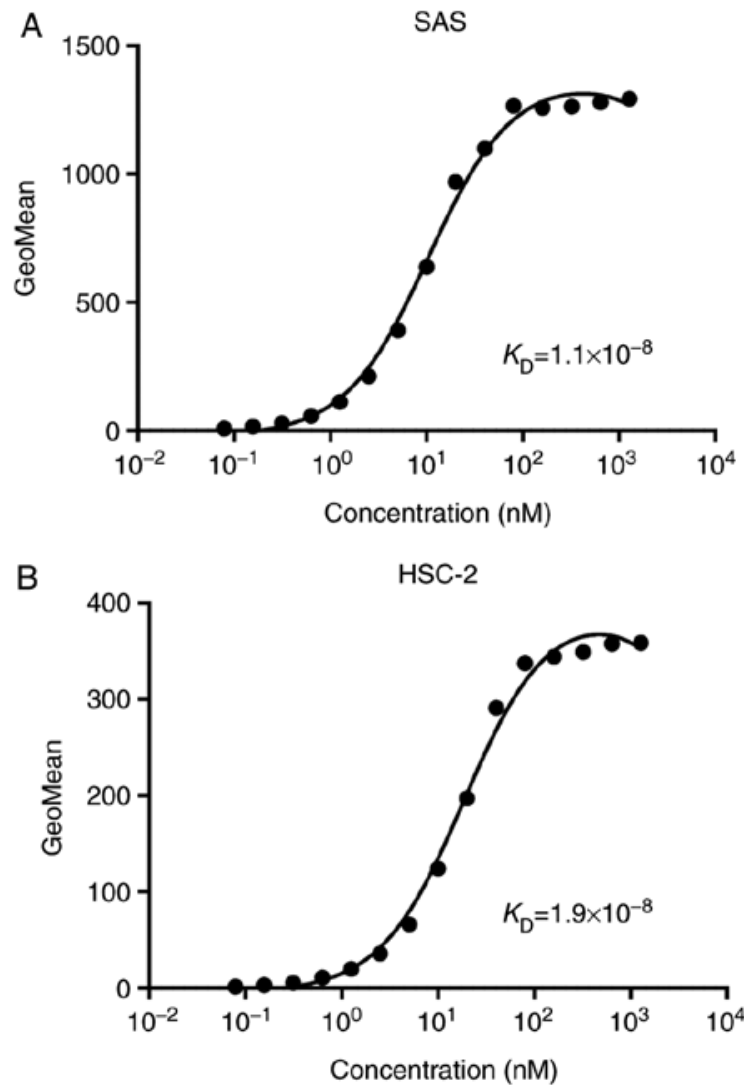

Figure 4. Determination of binding affinity of an anti-EpCAM $m A b$ for OSCC cells. (A) SAS and (B) HSC-2 cells were suspended in $100 \mu 1$ of serially diluted EpMab-16, followed by the addition of Alexa Fluor 488-conjugated anti-mouse IgG, and fluorescence data were collected. EpCAM, epithelial cell adhesion molecule; mAb, monoclonal antibody; OSCC, oral squamous cell carcinoma.
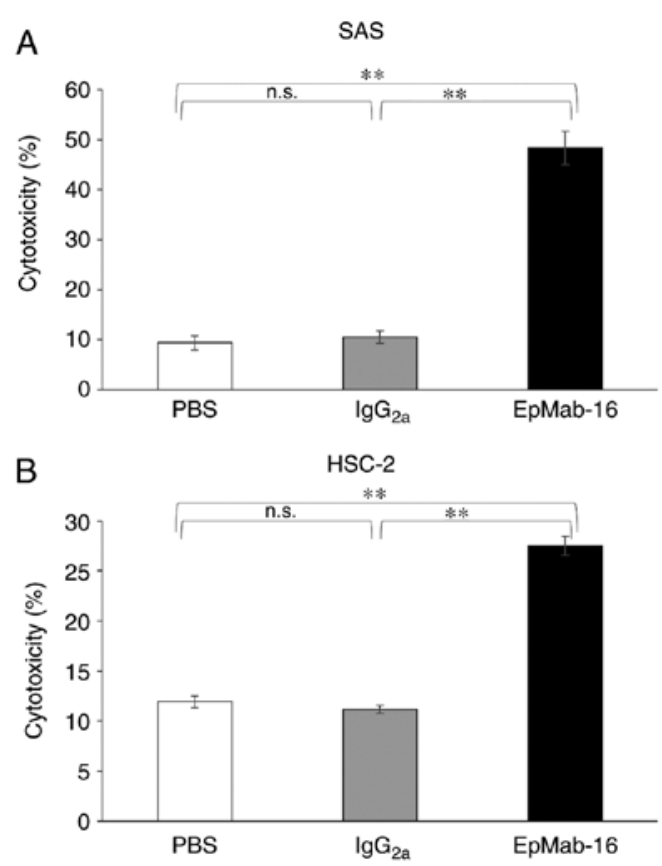

Figure 5. ADCC activity of an anti-EpCAM mAb in OSCC cells. ADCC activity of EpMab-16, control mouse $\operatorname{IgG}_{2 \mathrm{a}}$, and control PBS in (A) SAS and (B) HSC-2 cells. Values represent the mean \pm SEM. ${ }^{* *} \mathrm{P}<0.01$ (determined by ANOVA and Tukey's multiple comparisons tests). ADCC, antibody-dependent cellular cytotoxicity; EpCAM, epithelial cell adhesion molecule; mAb, monoclonal antibody; OSCC, oral squamous cell carcinoma; SEM, standard error of the mean; n.s., not significant. 

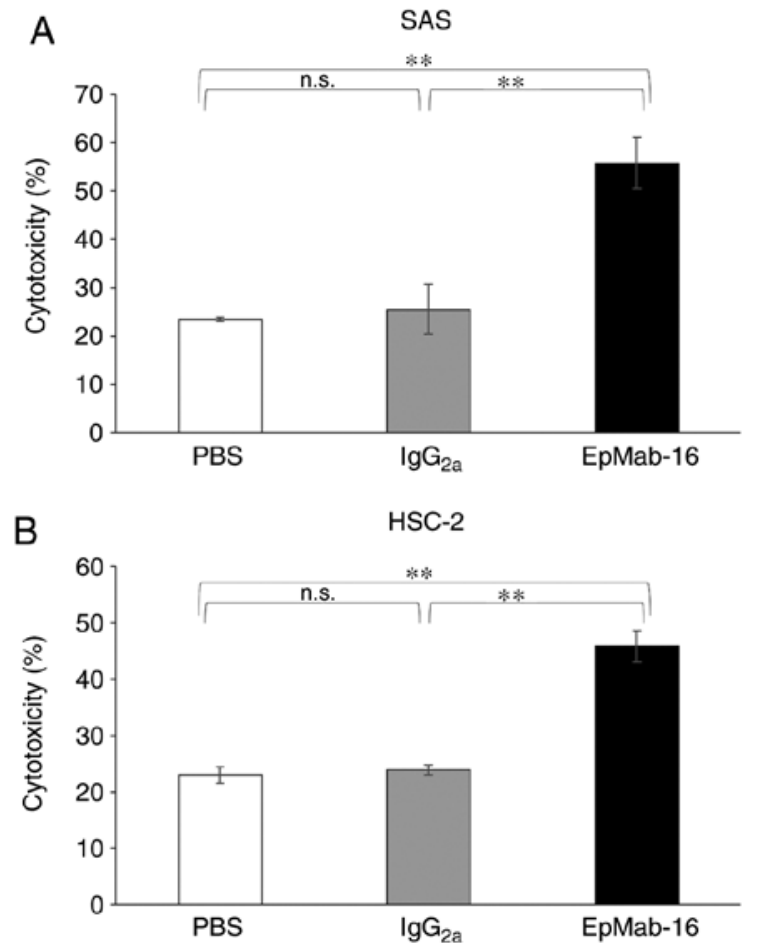

Figure 6. CDC activity of an anti-EpCAM $m A b$ in OSCC cells. CDC activity of EpMab-16, control mouse $\mathrm{IgG}_{2 \mathrm{a}}$, and control PBS in (A) SAS and (B) HSC-2 cells. Values represent the mean \pm SEM. ${ }^{* *} \mathrm{P}<0.01$ (determined by ANOVA and Tukey's multiple comparisons tests). CDC, complement-dependent cytotoxicity; EpCAM, epithelial cell adhesion molecule; mAb, monoclonal antibody; OSCC, oral squamous cell carcinoma; PBS, phosphate-buffered saline; SEM, standard error of the mean; n.s., not significant.

$\operatorname{IgG}_{2 \mathrm{a}}(11 \%$ cytotoxicity; $\mathrm{P}<0.01)$ or control PBS treatment $(12 \%$ cytotoxicity; $\mathrm{P}<0.01)$ (Fig. $5 \mathrm{~B})$.

EpMab-16 was also associated with more robust CDC activity ( $56 \%$ cytotoxicity) in SAS cells than control mouse $\operatorname{IgG}_{2 \mathrm{a}}$ ( $26 \%$ cytotoxicity; $\mathrm{P}<0.01)$ or control PBS treatment $(23 \%$ cytotoxicity; $\mathrm{P}<0.01$ ) (Fig. 6A). Similarly, EpMab-16-treated cells exhibited more CDC activity (46\% cytotoxicity) in HSC-2 cells than control mouse $\operatorname{IgG}_{2 \mathrm{a}}(24 \%$ cytotoxicity; $\mathrm{P}<0.01)$ or control PBS treatment (23\% cytotoxicity; $\mathrm{P}<0.01)$ (Fig. 6B). These favorable ADCC and CDC activity results indicated that EpMab-16 may induce strong antitumor action against oral cancer cells in vivo as well as in vitro.

EpMab-16 antitumor effect in mouse xenografts of SAS oral cancer cells. On days 1,8 , and 14 after SAS cell injections into the mice, EpMab-16 (100 $\mu \mathrm{g})$ or control mouse IgG (100 $\mu \mathrm{g})$ were injected i.p. in SAS xenograft model mice. Tumor formation was observed in mice from treatment and control groups. Tumor volume was measured on days 7, 10, 14, and 17 after SAS cell injection. EpMab-16-treated mice exhibited significantly less tumor growth on day $10(\mathrm{P}<0.05)$, day $14(\mathrm{P}<0.05)$ and day $17(\mathrm{P}<0.01)$ compared with IgG-treated control mice (Fig. 7A). Tumor volume reduction by EpMab-16 treatment was $37 \%$ as of day 17. Tumors from EpMab-16-treated mice weighed significantly less than tumors from IgG-treated control mice (20\% reduction, $\mathrm{P}<0.05$; Fig. 7B). Resected tumors on day 17 are presented in Fig. 7C. Total body weights did not significantly differ between the treatment and control groups (Fig. S1).
These results indicated that EpMab-16 reduced the growth of SAS xenografts, but did not altogether eliminate them.

EpMab-16 antitumor effect in mouse xenografts of HSC-2 oral cancer cells. On days 1, 8, and 14 after HSC-2 cell injections, EpMab-16 $(100 \mu \mathrm{g})$ or control mouse $\operatorname{IgG}(100 \mu \mathrm{g})$ were injected i.p. into HSC-2 xenograft model mice. Tumor formation was observed in mice in the treatment and control groups. Tumor volume was measured on days 7, 10, 14, and 18 after HSC-2 cell injection. The EpMab-16-treated mice exhibited significantly less tumor growth on day $18(\mathrm{P}<0.01)$ than the IgG-treated control mice (Fig. 8A). Tumor volume reduction by EpMab-16 treatment was 33\% as of day 18. Tumors from EpMab-16-treated mice weighed significantly less than tumors from IgG-treated control mice ( $21 \%$ reduction, $\mathrm{P}<0.05$; Fig. 8B). Resected tumors on day 18 are presented in Fig. 8C. Total body weights did not significantly differ between the treatment and control groups (Fig. S1). These results indicated that EpMab-16 reduced the growth of HSC-2 xenografts, although it did not eliminate them.

\section{Discussion}

EpCAM has been reported to be overexpressed in several cancers (23-27). EpCAM is overexpressed in $55-75 \%$ of ovarian cancers (24), and is overexpressed in over $90 \%$ of ovarian cancer metastatic lesions (25). Overexpression of EpCAM has been revealed to be correlated with poor prognosis, therapeutic failure and early tumor recurrence in hepatocellular carcinoma (HCC) patients (26). EpCAM has also been revealed to play important roles in proliferation, apoptosis, and metastasis during breast cancer progression (27). Therefore, EpCAM should be a promising novel therapeutic target.

In the present study, it was investigated whether anti-EpCAM mAbs could be useful for treating oral cancers via ADCC and CDC activity. We first developed a sensitive and specific anti-EpCAM mAb (EpMab-16, mouse $\operatorname{IgG}_{2 \mathrm{a}}$ ), which exhibited favorable potential in flow cytometry, western blot and immunohistochemical analyses. The present results also suggested that EpMab-16 had diagnostic efficacy in FFPE tissues because pathological diagnosis utilizes FFPE tissues. It was also demonstrated that EpMab-16 was associated with strong ADCC and CDC activity against SAS and HSC-2 OSCC cell lines in vitro. The ADCC and CDC activity of EpMab-16 against OSCC was more pronounced in SAS cells than HSC-2 cells, presumably because the EpCAM expression level and binding affinity are greater in SAS than in HSC-2. ADCC and CDC activities of EpMab-16 against Caco-2, a colon cancer cell line were also assessed, and values were 44 and $49 \%$, respectively (data not shown), suggesting that EpMab-16 could induce observable ADCC and CDC in other EpCAM-expressing cancers in addition to the oral cancer cell lines assessed in this study.

It was then investigated whether EpMab-16 exerted antitumor function against OSCC xenografts in vivo. Although EpMab-16 significantly reduced not only the growth of SAS and HSC-2 xenografts, but also the tumor weight of SAS and HSC-2 xenografts, the tumor reduction was not sufficient to eliminate them. We previously investigated whether 
A

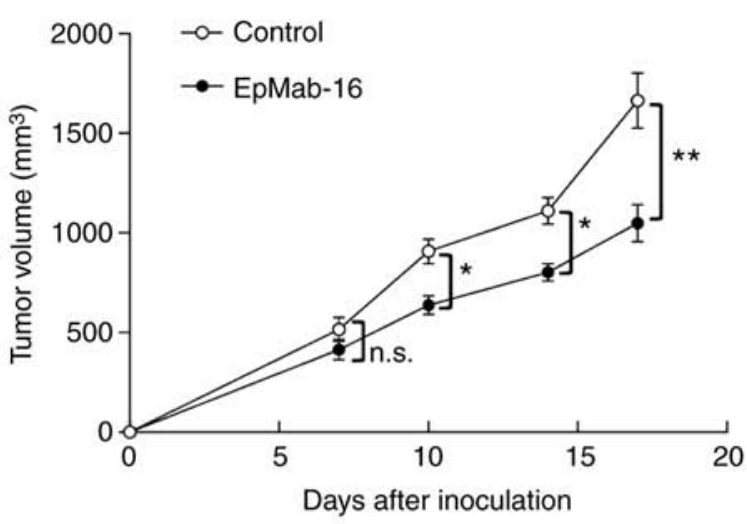

B

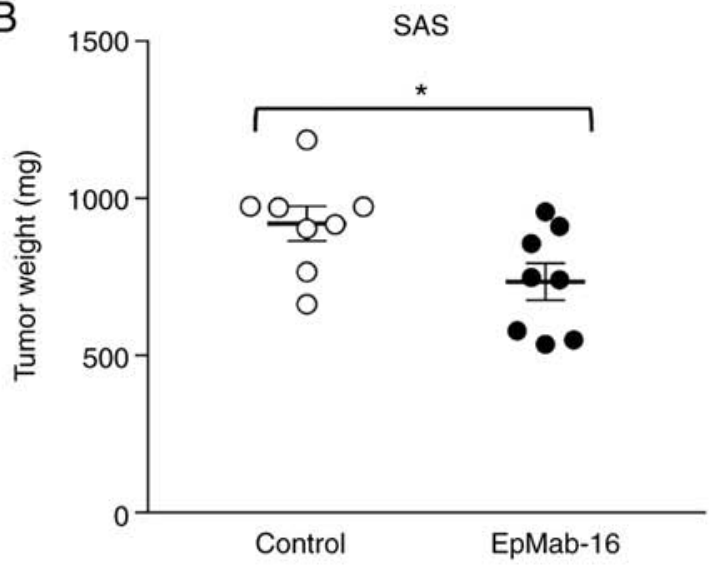

C

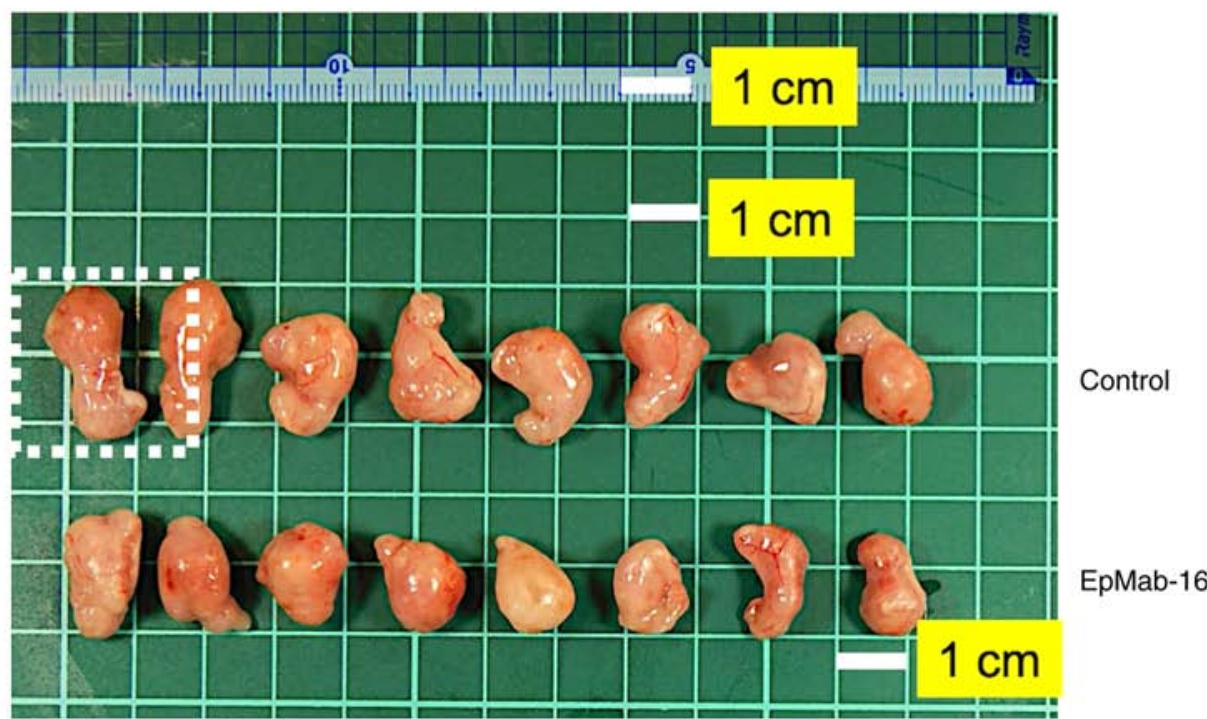

$\mathrm{D}$

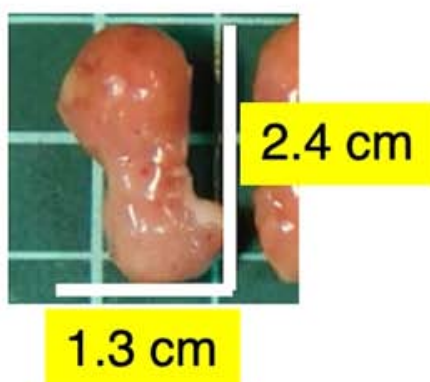

Figure 7. Antitumor activity of an anti-EpCAM mAb in SAS xenografts. (A) Tumor volume of SAS xenografts. SAS cells (5x $10^{6}$ cells) were injected subcutaneously into the left flank, and $100 \mu \mathrm{g}$ of EpMab-16 or control mouse IgG in $100 \mu \mathrm{l}$ PBS was injected i.p. into treatment and control mice, respectively. Additional antibodies were injected on days 8 and 14. The tumor volume was measured on days 7, 10, 14, and 17. Values represent the mean \pm SEM. ${ }^{*} \mathrm{P}<0.05$ and ${ }^{* *} \mathrm{P}<0.01$ (determined by ANOVA and Sidak's multiple comparisons tests) (B) Tumor weights of SAS xenografts. Tumors of SAS xenografts were resected from EpMab-16 and control mouse IgG groups. The tumor weight on day 17 was measured from excised xenografts. Values represent the mean \pm SEM. ${ }^{*} \mathrm{P}<0.05$ (determined by Welch's t-test). (C) Resected tumors of SAS xenografts from EpMab-16 and control mouse IgG groups on day 17. The tumor in the dotted region was the largest tumor in this experiment. The white square grids under the tumors are $1 \times 1 \mathrm{~cm}$ each. Scale bar, $1 \mathrm{~cm}$. (D) A magnified image of the largest tumor in the dotted region of C. The vertical and horizontal lengths for SAS cells were 2.4 and $1.3 \mathrm{~cm}$, respectively (estimated tumor volume, $2028 \mathrm{~mm}^{3}$ ). All mice possessed one tumor each. EpCAM, epithelial cell adhesion molecule; mAb, monoclonal antibody; PBS, phosphate-buffered saline; SEM, standard error of the mean; n.s., not significant.

podocalyxin (PODXL) could be a therapeutic target in OSCC using anti-PODXL mAbs developed by converting an anti-PODXL $\mathrm{mAb}$ of $\mathrm{IgG}_{1}$ subclass (PcMab-47) into a mouse $\mathrm{IgG}_{2 \mathrm{a}}$-type $\mathrm{mAb}\left(47-\mathrm{mG}_{2 \mathrm{a}}\right.$ ) to increase ADCC (28). This was further developed into $47-\mathrm{mG}_{2 \mathrm{a}} \mathrm{f}$, a core fucose-deficient variant of $47-\mathrm{mG}_{2 \mathrm{a}}$, also to increase its ADCC. In vivo analyses demonstrated that $47-\mathrm{mG}_{2 \mathrm{a}}-\mathrm{f}$, but not $47-\mathrm{mG}_{2 \mathrm{a}}$, exerted antitumor activity in SAS and HSC-2 xenograft models at a dose of $100 \mu \mathrm{g} / \mathrm{mouse} /$ week administered three times, indicating that a core fucose-deficient anti-PODXL $\mathrm{mAb}$ could be profitable for antibody-based therapy against PODXL-expressing OSCCs (28). We thereby anticipate that 
A

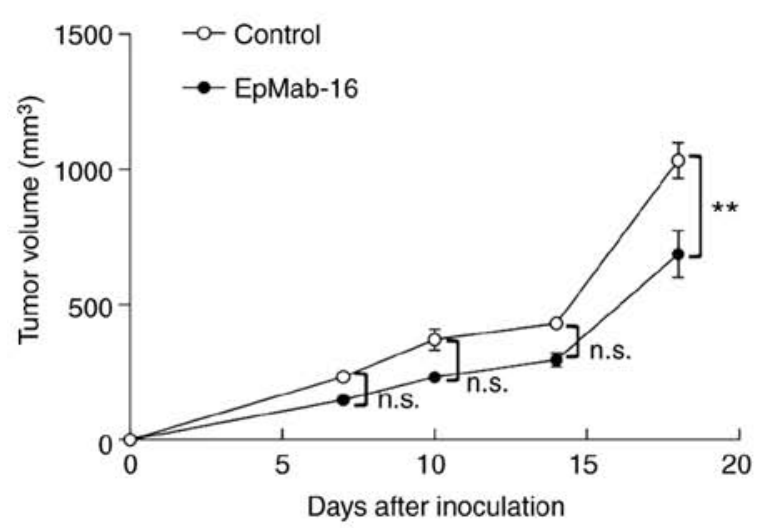

B

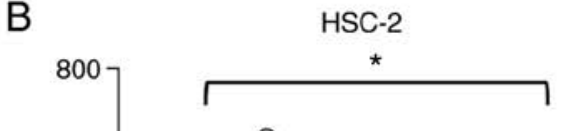

O

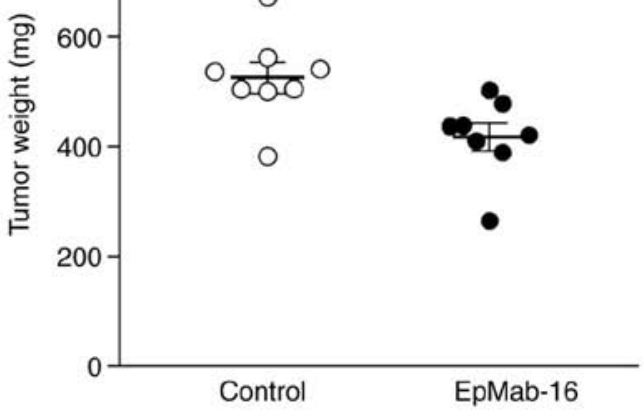

C

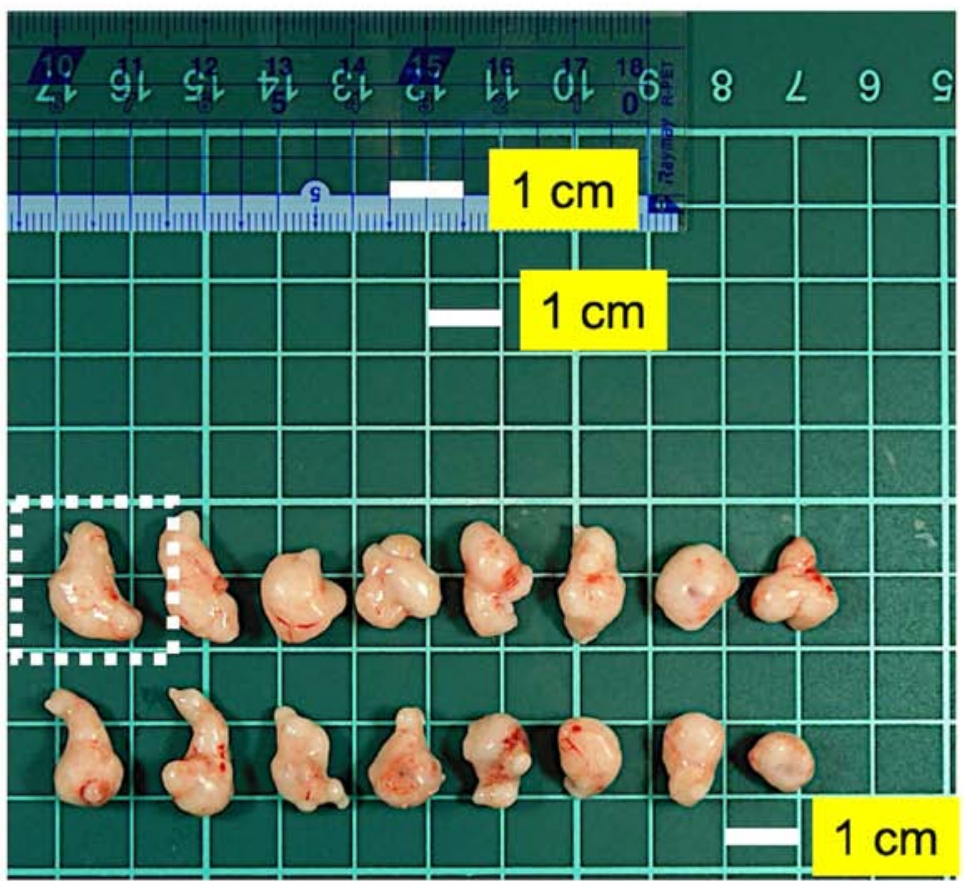

Control

D

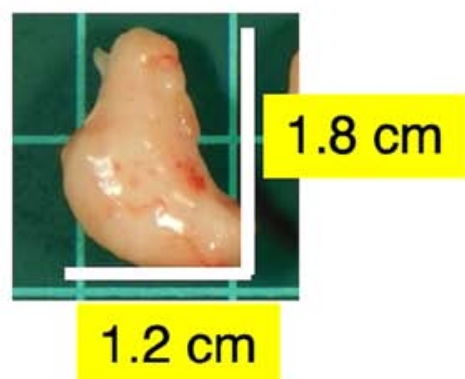

Figure 8. Antitumor activity of an anti-EpCAM mAb in HSC-2 xenografts. (A) Tumor volume of HSC-2 xenografts. HSC-2 cells (5x10 cells) were injected subcutaneously into the left flank, and $100 \mu \mathrm{g}$ of EpMab-16 or control mouse IgG in $100 \mu \mathrm{l}$ PBS was injected i.p. into treatment and control mice, respectively. Additional antibodies were then injected on days 8 and 14. The tumor volume was measured on days 7, 10, 14, and 18. Values represent the mean \pm SEM ${ }^{* *} \mathrm{P}<0.01$ (determined by ANOVA and Sidak's multiple comparisons tests). (B) Tumor weights of HSC-2 xenografts. Tumors of HSC-2 xenografts were resected from EpMab-16 and control mouse IgG groups. The tumor weight on day 18 was measured from excised xenografts. Values represent the mean \pm SEM. ${ }^{*} \mathrm{P}<0.05$ (determined by Welch's t-test). (C) Resected tumors of HSC-2 xenografts from EpMab-16 and control mouse IgG groups on day 18. The tumor in the dotted region was the largest tumor in this experiment. The white square grids under the tumors are $1 \mathrm{x} 1 \mathrm{~cm}$ each. Scale bar, $1 \mathrm{~cm}$. (D) A magnified image of the largest tumor in the dotted region of C. The vertical and horizontal lengths for HSC-2 cells were 1.8 and $1.2 \mathrm{~cm}$, respectively (estimated tumor volume, $1296 \mathrm{~mm}^{3}$ ). All mice possessed one tumor each. EpCAM, epithelial cell adhesion molecule; mAb, monoclonal antibody; PBS, phosphate-buffered saline; SEM, standard error of the mean; n.s., not significant.

developing a core fucose-deficient variant of EpMab-16 will reveal a similar enhancement of ADCC activity in a future study.
Results of this study and our previous work, which includes the development of mAbs effective in SAS and HSC-2 xenografts against epidermal growth factor receptor (EGFR) 
(clone EMab-17, mouse $\operatorname{IgG}_{2 \mathrm{a}}$ ) (29), human epidermal growth factor receptor 2 (HER2) (clone $\mathrm{H}_{2} \mathrm{Mab}-19$, mouse $\mathrm{IgG}_{2 \mathrm{~b}}$ ) (30), and cancer-specific mAb (CasMab) against podoplanin (PDPN) (31), suggest that the targeting of several molecules, such as PODXL, EGFR, HER2, PDPN, as well as EpCAM, could be an effective therapy to cure OSCCs. In the future, cancer-specific anti-EpCAM mAbs may also be developed that can reduce the adverse effects of traditional antibody therapy.

\section{Acknowledgements}

We would like to thank Ms. Saori Handa, Ms. Saki Okamoto, and Mr. Yu Komatsu (Department of Antibody Drug Development, Tohoku University Graduate School of Medicine) for technical assistance of in vitro experiments, and Ms. Akiko Harakawa (Institute of Microbial Chemistry (BIKAKEN), Numazu, Microbial Chemistry Research Foundation) for technical assistance of animal experiments.

\section{Funding}

This research was supported in part by the Japan Agency for Medical Research and Development (AMED) under grant nos. JP20am0401013 (to YK), JP20am0101078 (to YK), and JP20ae0101028 (to YK), and by the Japan Society for the Promotion of Science (JSPS) Grants-in-Aid for Scientific Research (KAKENHI) grant no. 17K07299 (to MKK), grant nos. 19K07705 (to YK), and 20K16322 (to MS).

\section{Availability of data and materials}

The datasets used and/or analyzed during the study are available from the corresponding author on reasonable request.

\section{Authors' contributions}

HHo, JT, TO, TN, MS TA, YS, and MY performed the experiments. MKK analyzed the experimental data. MK, HHa, and YK designed the present study and wrote the manuscript. All the authors read and approved the final manuscript.

\section{Ethics approval and consent to participate}

Animal studies for ADCC and the antitumor activity were approved by the Institutional Committee for experiments of the Institute of Microbial Chemistry (Permit no. 2019-066).

\section{Patient consent for publication}

Not applicable.

\section{Competing interests}

The authors declare that they have no competing interests.

\section{References}

1. Okegawa T, Pong RC, Li Y and Hsieh JT: The role of cell adhesion molecule in cancer progression and its application in cancer therapy. Acta Biochim Pol 51: 445-457, 2004.
2. Petruzzelli L, Takami M and Humes HD: Structure and function of cell adhesion molecules. Am J Med 106: 467-476, 1999.

3. Maheshwari G, Brown G, Lauffenburger DA, Wells A and Griffith LG: Cell adhesion and motility depend on nanoscale RGD clustering. J Cell Sci 113: 1677-1686, 2000.

4. Trzpis M, McLaughlin PM, de Leij LM and Harmsen MC Epithelial cell adhesion molecule: More than a carcinoma marker and adhesion molecule. Am J Pathol 171: 386-395, 2007.

5. Herlyn M, Steplewski Z, Herlyn D and Koprowski H: Colorectal carcinoma-specific antigen: Detection by means of monoclonal antibodies. Proc Natl Acad Sci USA 76: 1438-1442, 1979.

6. Imrich S, Hachmeister M and Gires O: EpCAM and its potential role in tumor-initiating cells. Cell Adh Migr 6: 30-38, 2012.

7. Sen $\mathrm{S}$ and Carnelio S: Expression of epithelial cell adhesion molecule (EpCAM) in oral squamous cell carcinoma. Histopathology 68: 897-904, 2016.

8. Baeuerle PA and Gires O: EpCAM (CD326) finding its role in cancer. Br J Cancer 96: 417-423, 2007.

9. Went P, Vasei M, Bubendorf L, Terracciano L, Tornillo L, Riede U, Kononen J, Simon R, Sauter G and Baeuerle PA: Frequent high-level expression of the immunotherapeutic target Ep-CAM in colon, stomach, prostate and lung cancers. Br J Cancer 94: 128-135, 2006.

10. Bray F, Ferlay J, Soerjomataram I, Siegel RL, Torre LA and Jemal A: Global cancer statistics 2018: GLOBOCAN estimates of incidence and mortality worldwide for 36 cancers in 185 countries. CA Cancer J Clin 68: 394-424, 2018.

11. Tota JE, Anderson WF, Coffey C, Califano J, Cozen W, Ferris RL, St John M, Cohen EE and Chaturvedi AK: Rising incidence of oral tongue cancer among white men and women in the United States, 1973-2012. Oral Oncol 67: 146-152, 2017.

12. Hussein AA, Helder MN, de Visscher JG, Leemans CR, Braakhuis BJ, de Vet HCW and Forouzanfar T: Global incidence of oral and oropharynx cancer in patients younger than 45 years versus older patients: A systematic review. Eur J Cancer 82: 115-127, 2017.

13. Rivera C: Essentials of oral cancer. Int J Clin Exp Pathol 8: 11884-11894, 2015.

14. Güneri P and Epstein JB: Late stage diagnosis of oral cancer: Components and possible solutions. Oral Oncol 50: 1131-1136, 2014.

15. Vokes EE: Induction chemotherapy for head and neck cancer: Recent data. Oncologist 15 (Suppl 3): S3-S7, 2010.

16. Marcazzan S, Varoni EM, Blanco E, Lodi G and Ferrari M: Nanomedicine, an emerging therapeutic strategy for oral cancer therapy. Oral Oncol 76: 1-7, 2018.

17. Furness S, Glenny AM, Worthington HV, Pavitt S, Oliver R, Clarkson JE, Macluskey M, Chan KK and Conway DI: Interventions for the treatment of oral cavity and oropharyngeal cancer: Chemotherapy. Cochrane Database of Systematic Reviews: CD006386, 2011.

18. Itai S, Fujii Y, Nakamura T, Chang YW, Yanaka M, Saidoh N, Handa S, Suzuki H, Harada H, Yamada S, et al: Establishment of CMab-43, a sensitive and specific anti-CD133 monoclonal antibody, for immunohistochemistry. Monoclon Antib Immunodiagn Immunother 36: 231-235, 2017.

19. Ota T, Suzuki Y, Nishikawa T, Otsuki T, Sugiyama T, Irie R, Wakamatsu A, Hayashi K, Sato H, Nagai K, et al: Complete sequencing and characterization of 21,243 full-length human cDNAs. Nat Genet 36: 40-45, 2004.

20. Otsuki T, Ota T, Nishikawa T, Hayashi K, Suzuki Y, Yamamoto J, Wakamatsu A, Kimura K, Sakamoto K, Hatano N, et al: Signal sequence and keyword trap in silico for selection of full-length human cDNAs encoding secretion or membrane proteins from oligo-capped cDNA libraries. DNA Res 12: 117-126, 2005.

21. Kimura K, Wakamatsu A, Suzuki Y, Ota T, Nishikawa T, Yamashita R, Yamamoto J, Sekine M, Tsuritani K, Wakaguri $\mathrm{H}$, et al: Diversification of transcriptional modulation: Large-scale identification and characterization of putative alternative promoters of human genes. Genome Res 16: 55-65, 2006.

22. Itoh M, Yasunishi A, Imamura K, Kanamori-Katayama M, Suzuki H, Suzuki M, Carninci P, Kawai J and Hayashizaki Y: Constructing ORFeome resources with removable termination codons. Biotechniques 41: 44, 46, 48 passim, 2006.

23. Jing Y, Zhou L, Chen J, Xu H, Sun J, Cai M, Jiang J, Gao J and Wang H: Quantitatively mapping the assembly pattern of EpCAM on cell membranes with peptide probes. Anal Chem 92: $1865-1873,2020$

24. Vorobyeva A, Konovalova E, Xu T, Schulga A, Altai M, Garousi J, Rinne SS, Orlova A, Tolmachev V and Deyev S: Feasibility of imaging EpCAM expression in ovarian cancer using radiolabeled DARPin Ec1. Int J Mol Sci 21: 3310, 2020. 
25. van den Brand D, van Lith SAM, de Jong JM, Gorris MAJ, Palacio-Castañeda V, Couwenbergh ST, Goldman MRG, Ebisch I, Massuger LF, Leenders WPJ, et al: EpCAM-binding DARPins for targeted photodynamic therapy of ovarian cancer. Cancers (Basel) 12: 1762, 2020.

26. Pandit H, Li Y, Zheng Q, Guo W, Yu Y, Li S and Martin RCG: Carcinogenetic initiation contributed by EpCAM+ cancer cells in orthotopic HCC models of immunocompetent and athymic mice. Oncotarget 11: 2047-2060, 2020.

27. Zhang D, Yang L, Liu X, Gao J, Liu T, Yan Q and Yang X: Hypoxia modulates stem cell properties and induces EMT through N-glycosylation of EpCAM in breast cancer cells. J Cell Physiol 235: 3626-3633, 2020.

28. Itai S, Ohishi T, Kaneko MK, Yamada S, Abe S, Nakamura T, Yanaka M, Chang YW, Ohba SI, Nishioka Y, et al: Anti-podocalyxin antibody exerts antitumor effects via antibody-dependent cellular cytotoxicity in mouse xenograft models of oral squamous cell carcinoma. Oncotarget 9: 22480-22497, 2018.
29. Takei J, Kaneko MK, Ohishi T, Kawada M, Harada H and Kato Y: A novel anti-EGFR monoclonal antibody (EMab-17) exerts antitumor activity against oral squamous cell carcinomas via antibody-dependent cellular cytotoxicity and complement-dependent cytotoxicity. Oncol Lett 19: 2809-2816, 2020.

30. Takei J, Kaneko MK, Ohishi T, Kawada M, Harada H and Kato Y: $\mathrm{H}(2)$ Mab-19, an anti-human epidermal growth factor receptor 2 monoclonal antibody exerts antitumor activity in mouse oral cancer xenografts. Exp Ther Med 20: 846-853, 2020.

31. Kaneko MK, Nakamura T, Kunita A, Fukayama M, Abe S, Nishioka Y, Yamada S, Yanaka M, Saidoh N, Yoshida K, et al: ChLpMab-23: Cancer-specific human-mouse chimeric anti-podoplanin antibody exhibits antitumor activity via antibody-dependent cellular cytotoxicity. Monoclon Antib Immunodiagn Immunother 36: 104-112, 2017.

(i) $\Theta$ This work is licensed under a Creative Common Attribution-NonCommercial-NoDerivatives 4.0 International (CC BY-NC-ND 4.0) License. 